\title{
Réparation juridique du préjudice sexuel dans les ruptures traumatiques de l'urètre
}

\author{
Sylla C., Ndoye A., Ba M., Diagne B..A., Sylla S. \\ Université Cheikh Anta Diop de Dakar - Faculté de Médecine, Pharmarcie \\ et Odonto-Stomatologie - Dakar (Sénégal)
}

\section{RESUME}

\begin{abstract}
Après rupture traumatique de l'urètre postérieur, le préjudice sexuel peut être retenu et évalué selon certains critères parmi lesquels les résultats des injections intracaverneuses méritent d'être discutés. Seul le préjudice certain et direct est indemnisable ce qui nécessite de la part de l'expert un examen méthodique et minutieux.
\end{abstract}

Mots clés : urètre, traumatisme, préjudice

L'aspect sexuel des conséquences des traumatismes de l'urètre postérieur est souvent sous estimé. La stérilité et les troubles de l'érection sont possibles et liés au siège de la rupture urétrale mais aussi au traumatisme chirurgical lors de la réparation des lésions. L'impuissance surviendrait dans 30 à $80 \%$ des cas [6] en rapport avec les lésions vasculaires ou nerveuses. Pour certains auteurs la moitié de ces impuissances serait temporaire, la récupération étant possible jusqu'à 2 ans après le traumatisme. Gibson [4] rapportant une série de 35 patients présentant une impuissance post fracturaire observe une impuissance totale à 3 ans dans $37 \%$ des cas, et une impuissance temporaire jusqu'à 19 mois dans $21 \%$ des cas. La part psychique est importante mais non exclusive car ce serait négliger les lésions loco-régionales liées au traumatisme ou aux interventions chirurgicales souvent délabrantes vis à vis des structures impli- quées dans l'érection. Si le préjudice sexuel est retenu l'expert devra en évoquer toutes les possibilités thérapeutiques [1].

Lors de la réparation juridique d'un dommage corporel l'expert doit garder à l'esprit tout au long de l'expertise qu'un préjudice ne sera indemnisé qu'à la double condition d'être certain et de pouvoir être rapporté d'une manière certaine et directe au fait générateur de responsabilité. Lorsque le préjudice allégué est simplement fonctionnel ne se traduisant à l'examen par aucun signe clinique spécifique, cas des troubles de la libido et de l'impuissance, et même si l'expert affirme la réalité du préjudice, le magistrat peut refuser toute indemnisation [7]. C'est pourquoi dans le cadre du préjudice sexuel après rupture traumatique de l'urètre, il incombe à l'expert de le décrire et de rechercher tous les éléments qui conduisent à une certitude diagnostique. Parmi ces éléments les résultats des tests d'érection provoquée par une drogue vaso-active doivent être pris en compte tant pour la certitude diagnostique que pour le pronostic à long terme intervenant dans l'indemnisation.

Notre étude s'intéresse à la réparation juridique du préjudice sexuel dans les ruptures traumatiques de l'urètre postérieur .

\section{IMPUTABILITE}

Cette question fait discuter les différents critères énoncés par Muller et Cordonnier. 
L'étude de l'ensemble de ces critères permet d'établir l'imputabilité du traumatisme par rapport aux plaintes alléguées par le patient.

\section{La plausibilité pathogénique}

Elle a été soulignée par Menelec [8] et impose une analyse méthodique, minutieuse pour chaque cas, le patient entendant imputer l'ensemble de ses troubles au traumatisme urétral. Une filiation logique scientifiquement conforme aux lois de la pathogénie et de la physiopathologie doit être exigée. Les troubles sexuels sont plus délicats à admettre, le mécanisme physiologique devant prouver en outre la filiation lésion urétrale, nerveuse ou vasculaire [2].

\section{La certitude diagnostique}

Elle est indispensable à établir. On ne peut indemniser qu'un préjudice certain et non pas seulement allégué. Il s'agit parfois d'un problème délicat car un certain nombre de troubles ne peuvent être ni prouvés ni vérifiés lors de l'expertise. L'expert appuie sa discussion sur l'ensemble des données recueillies mais reste limité dans les examens complémentaires à demander car ces derniers ne doivent avoir ni un caractère agressif ou dangereux ni un caractère franchement désagréable.

\section{L'intégrité préalable}

Elle introduit la notion d'état antérieur. Il est nécessaire de préciser l'existence ou non d'antécédents urologiques. L'expert peut se mettre en rapport avec "toute personne informée" tout en ne dérogeant pas aux principes du secret médical. Si le patient refuse qu'une information soit transmise, ce refus vaudra présomption contre lui. La connaissance de cet état antérieur et son évaluation donneront la mesure de ce que le responsable doit indemniser.

\section{La concordance de siège}

L'argument topographique est déterminant car le siège membraneux ou membrano- prostatique de la lésion signe l'origine traumatique [3].

\section{Le délai d'apparition des troubles}

Lorsque ce délai est court le traumatisme apparait aisément compatible avec les plaintes du patient, dans le cas contraire il faut rechercher une continuité évolutive.

\section{La continuité évolutive}

Elle est déterminante lorsque le délai d'apparition des troubles est long. Néanmoins compte tenu du génie évolutif des lésions urétrales l'expert doit être prudent avant d'écarter l'imputabilité par ce paramètre tout comme il doit en tenir compte en cas de lésion antérieure à l'accident.

\section{La réalité du traumatisme}

Les données nécessaires à cette analyse sont apportées par l'étude du dossier comportant les certificats médicaux, les différents compte-rendus d'hospitalisation et les examens complémentaires (urographie intraveineuse, urétrocystographie).

\section{LE PREJUDICE SEXUEL}

Le préjudice sexuel est différent de l'IPP qui, elle, est certaine, quantifiable et définitive. Il se définit comme l'impossibilité totale ou partielle où se trouve la victime, du fait des séquelles traumatiques qu'elle présente, soit d'accomplir l'acte sexuel, soit de procréer ou de se reproduire d'une manière normale. Menelec [7] souligne la différence qui existe entre préjudice sexuel, préjudice d'agrément et préjudice moral. Le préjudice d'agrément est défini par l'impossibilité où se trouve la victime du fait de l'altération traumatique de ses capacités fonctionnelles de s'adonner à certaines activités culturelles, sportives ou de loisir. Seule la conjointe peut prétendre à la réparation d'un préjudice moral 


\section{Conduite de l'expertise dans le cadre du préjudice sexuel}

Compte tenu de l'aspect parfois délicat du sujet, l'expert doit pouvoir aborder cette rubrique dans un climat de confiance. Le but de l'examen sera de confirmer l'existence de ce préjudice et de savoir s'il est permanent, améliorable ou définitif.

\section{a) L'interrogatoire représente le temps essentiel de l'examen :}

- circonstances de l'accident et évolution à la recherche d'éléments physiques et affectifs susceptibles d'être impliqués dans la vie sexuelle du sujet,

- doléances spontanément exprimés portant sur la vie sexuelle,

- questions spécifiques à poser ou éventuellement recueillies par l'intermédiaire d'un "sapiteur". Les échecs doivent pouvoir être racontés de même que toutes les thérapeutiques tentées jusqu'à présent,

- noter les souhaits formulés dans le domaine de la fécondité mais aussi sur la fécondité elle même

Rousseau [10] propose d'interroger la partenaire en présence et en l'absence du sujet. Ceci est parfois délicat mais il incombe à chaque partie de prouver les faits nécessaires au succès de sa prétention.

b) L'examen physique en dehors de l'examen général s'intéressera avec minutie aux caractères sexuels primaires, secondaires, et à l'examen neurologique.

Du point de vue des examens complémentaires l'expert pourra demander à se faire communiquer des examens déjà pratiqués (rigidimétrie, examen doppler des vaisseaux péniens ...). Dans le cadre de l'expertise certains examens peuvent être demandés à condition qu'ils soient strictement atraumatiques.

\section{c) Le préjudice est-il améliorable?}

De nos jours, les résultats des tests d'érection par injection intracaverneuse occupent une place importante dans l'évaluation de ces troubles et de leur pronostic à plus ou moins long terme. Ces résultats sont à interpréter en fonction du contexte psychogène toujours présent. Les drogues vaso actives les plus fréquemment utilisées sont les prostaglandines, les alpha bloquants et la papavérine. Il importera de préciser le traitement actuel de cette impuissance (drogues vaso actives, vacuum) et si l'indication d'une prothèse pénienne ou d'une chirurgie vasculaire a été discutée.

Il s'agit en fait d'une expertise difficile nécessitant souvent l'avis de spécialistes.

\section{Evaluation du préjudice sexuel}

Une fois que l'expert affirme le caractère certain du préjudice, ainsi que le caractère certain et direct de la relation causale, son rôle sera de décrire le plus exactement le préjudice en le situant dans son contexte (âge du sujet, situation matrimoniale, nombre d'enfants existant ...). Les troubles peuvent être qualifiés en fonction des échelles classiques affectées au préjudice esthétique (modéré, important ... ou encore $5 / 7,6 / 7$...). C'est au magistrat, et à lui seul, éclairé par les descriptions précises de l'expert , qu'il incombe d'évaluer la "réparation“. Néanmoins cet aspect est discuté par Rousseau [10] qui soulève le caractère intime et personnel des notes relevées au cours de l'expertise et qui dans la pure tradition du secret médical sont difficilement mentionnées en totalité dans un rapport d'expertise.

Bien que le préjudice sexuel ne donne en principe lieu à aucune I.P.P certains auteurs ont proposé un barème pour le dommage génito sexuel en fixant un taux de déficit physiologique et en donnant une description situationnelle $[9,10]$. A ces taux variables de 0 à $40 \%$ s'ajoutent le handicap qui est l'impossibilité ou l'incapacité de faire face à une situation donnée. Pour un même taux de déficit physiologique ce handicap sera différent pour : 
- le sujet jeune ou âgé,

- en fonction des habitudes antérieures,

- en fonction des souhaits d'avoir un ou plusieurs enfants.

Pour notre part nous pensons que dans le cadre de l'impuissance il est important de pouvoir préciser :

- la sensibilité aux médications intracaverneuses (drogues vaso-actives),

- le choix thérapeutique du patient,

- l'éventuelle indication d'une chirurgie vasculaire ou de l'implantation d'une prothèse pénienne.

L'impuissance des traumatismes urétraux est souvent d'origine psychogène ou nerveuse en conséquence des interventions chirurgicales. Cette impuissance est généralement sensible aux droques vasoactives en injections intra caverneuses (IIC) et il nous paraît important dans le cadre de l'indemnisation de distinguer trois groupes :

- Groupe 1 : sujets non sevrable des IIC (lésions nerveuses importantes),

- Groupe 2 : sujets guéris après sevrage des IIC (origine psychogène le plus souvent),

- Groupe 3 : sujets ne répondant pas aux drogues vaso actives en IIC posant ainsi le problème des prothèses péniennes.

Il s'agit là d'autant d'éléments que le juge sera amener à prendre en compte dans le cadre de l'indemnisation du préjudice sexuel.

Chez l'enfant les séquelles génitales éventuelles sont à juger après la puberté, ce que l'expert ne doit pas manquer de préciser dans son rapport.

\section{Indemnisation de la conjointe}

Cette indemnisation peut être accordée au titre du préjudice moral à condition que ce préjudice soit personnel et distinct de celui éprouvé par la victime et qu'il ait un caractère exceptionnel [2].

\section{CONCLUSIONS}

Face aux séquelles urinaires et génitales des ruptures traumatiques de l'urètre postérieur, le médecin expert est confronté à une situation où il ne devra être ni trop optimiste ni trop pessimiste. Certains patients présentant une impuissance peuvent récupérer une fonction sexuelle normale. Le préjudice sexuel lorsqu'il est retenu doit donner lieu à une description la plus exacte possible afin que le magistrat correctement éclairé puisse indemniser le patient. Une fois démontré il doit être décrit minutieusement en précisant son caractère améliorable ou non. Une avancée significative de la recherche dans le domaine du traitement per os des troubles de l'érection modifiera probablement l'approche thérapeutique de ces troubles (5). Des réserves sont à émettre quant à l'avenir du patient du fait de la dégradation possible des résultats thérapeutiques.

\section{REFERENCES}

1. AUBERT J., AUBERT M.N., IRANI J. : L'impuissance dans le cadre des fractures du bassin. A pro pos de 16 observations. Prog. Uro. 1992, 2, 635-644.

2. BENNANI S., BENJELLOUN S. : Répartition juridique des traumatismes de l'urètre postérieur chez l'homme. J. Urol. 1994, 4, 217-222.

3. FIQUET J.M., LOZE J.F., HERMANOWICZ M., RICHAUD C. : Appréciation du dommage au cours des traumatismes de l'urèthre postérieur. Rev. Franç. D.C., 1981, 7, 4, 425-428.

4. GIBSON G.R. : Impotence following fractured pelvis and ruptured urethra. Br. J. Urol., 1970, 42, 86-88.

5. GIULIANO F. : Le traitement per os des troubles de l'érection. Prog. Uro. 1997, 7, 108-109.

6. LE GUILLOU M., FERRIERE J.M. : Traumatisme de l'urètre postérieur. Encycl. Med. Chir. Rein, Organes génito-urinaires, 1983, 77, 18340, 2.

7. MELENEC L. : Le préjudice sexuel. Rev. Franç. D.C., 1978, 4, 1, 31-38.

8. MELENNEC L., KOURILSKY O., LASSAU J.P., MATHIEU F. : In :Evaluation du handicap et du dommage corporel - Barème international des invalidités Paris, Masson, 1991, 287-306. 
9. PAPELARD A. : Comment évaluer le dommage génito-sexuel ? 4e JERM, Paris-Bobigny : Masson, 1988 : 297-298.

10. ROUSSEAU C., FOURNIER C., BLANC-MOLLET M., CARBONIE C. : L'évaluation du dommage atteignant la fonction de reproduction. Rev. Franç. D.C., 1986, 12, 1, 87-94.

\section{ABSTRACT}

Legal compensation of sexual prejudice after traumatic urethral rupture

$$
\text { C. SYLLA et al. }
$$

After traumatic posterior urethral rupture, sexual prejudice can be evaluated with some criteria among which results of intracavernous injections must be discussed. The check up of the medical expert must be methodic and meticulous because only direct prejudice is compensable.

Key words : urethra, traumatism, prejudice. 